\title{
An Investigation on an Optimal Control Problem with a Fractional Constraint in the Riemann-Liouville Sense
}

\author{
Mehdi Nategh $^{1, \mathrm{a}}$, Bahram Agheli ${ }^{2, \mathrm{~b} *}$ \\ ${ }^{1}$ Department of Mathematics, University of Mazandaran, Babolsar, Iran \\ 2 Department of Mathematics, Qaemshahr Branch, Islamic Azad University, Qaemshahr, Iran \\ a m.nateghp@yahoo.com, b b.agheli@qaemshahriau.ac.ir \\ *b.agheli@qaemiau.ac.ir
}

Keywords: Riemann-Liouville optimal control problem, Volterra integro-differential equation, Caputo fractional derivative.

Abstract. This work, deals with a fractional optimal control problem (in the Riemann-Liouville sense). An analytic description of an initial value appears in the constraint of the optimal control problem is presented and some sufficient and necessary conditions for the given initial value is obtained. Making use of an auxiliary variable together with the optimal control law, the given problem is converted into a system of ordinary integro-differential equations. Then using the Adomian decomposition method, an approximate solution is illustrated.

\section{Introduction}

The present work concerns about an optimal control problem of non-integer order introduced by extremizing the functional

$$
J(x)=\frac{1}{2} \int_{a}^{b}\left(x^{2}(t)+u^{2}(2)+f(t) x(t)+g(t) u(t)\right) d t
$$

subject to the following initial value problem

$$
\left\{\begin{array}{l}
D_{a^{+}}^{\alpha} x(t)=a(t) x(t)+b(t) u(t)+\int_{a}^{t} K(t, s) \phi(x(s)) d s \\
x(a)=x_{a}, I_{a^{+}}^{1-\alpha} x\left(a^{+}\right)=0,
\end{array}\right.
$$

where $D_{a^{+}}^{\alpha}$ and $I_{a^{+}}^{1-\alpha}$ are fractional differential of order $\alpha$ and fractional integral of order $1-\alpha$ respectively.

This problem is a fractional version of the problem given in [1] by

$$
J(x)=\int_{a}^{b}\left(x^{2}(t)+u^{2}(2)+f(t) x(t)+g(t) u(t)\right) d t
$$

subject to the following initial value problem

$$
\left\{\begin{array}{l}
x^{\prime}(t)=a(t) x(t)+b(t) u(t)+\int_{a}^{t} K(t, s) \phi(x(s)) d s \\
x(a)=x_{a}
\end{array}\right.
$$

where, an approximate solution by the Legendre wavelets and collocation methods are obtained. 
Non-integer order dynamics in the both local and non-local cases, practically has been proven to be a breakthrough in the study of some dark corners of the rapidly thriving modern sciences $[2,3,4]$. Some intricate mechanisms those are governed by the power law, are studied numerically and analytically in terms of local or non-local fractional dynamics, and it has been done with higher level of precision than the classical models $[5,6]$.

Optimal control problems involving Volterra integro-differential systems of ordinary type, i.e., involving ordinary differential operator, has a rich history. In general, an integro-differential equation governs an evolution of a state $x(t)$ through time for which, the rate of change is proportioned to a time average. Such evolutions are observed naturally in a wide research areas such as population dynamics and elasticity of Kirchhoff type.

Since the optimal control problems essentially deal with some palpable level of applications, and due to the urgent needs of the calculations in physical and engineering, the numerical studies and solution techniques are as important as the existence and uniqueness issues. However, in non-integer order problems in the sense of singular differential operators, the numerical investigations demands special care while the singularity of a kernel in a definition of some fractional operators, could not be handled by many classical theorems. In this way, some new approaches and techniques are introduced (see for example [7, 8]).

In the present work, using a change of variable based on Caputo derivative, a Riemann-Liouville equation is converted into an ordinary integro-differential equation. This conversion simplifies the calculations and the problem takes a classical form. However, the singularity still remains but in the sense of Caputo.

The work is organized as follows:

The next section is devoted to a background for non-integer order calculus and it also provides a discussion on the concept of initial value of fractional integral form which is introduced in the problem (2). In section 3, we utilize an auxiliary variable in term of Caputo differential operator to convert (2) into a a problem which involves an ordinary differential operator. Section 4 deals with a numerical illustration.

\section{Arbitrary order calculus: preliminaries and discussions}

(See $[9,10]$ for some history notes and more detailed discussions.)

Defination 1. Let $[a, b]$ be an interval and $x$ be an integrable function. The Riemann-Liouville fractional integral $I_{a^{+}}^{\alpha} x$ and derivative $D_{b^{-}}^{\alpha} x$ of order $\left.n-1<\alpha \leq n\right)$ are defined by

$$
\begin{aligned}
I_{a^{+}}^{\alpha} x(t) & =\frac{1}{\Gamma(\alpha)} \int_{a}^{t} \frac{x(\tau)}{(t-\tau)^{1-\alpha}} d \tau \quad \text { a.e }(a, b], \\
D_{a^{+}}^{\alpha} x(t) & =\frac{1}{\Gamma(1-\alpha)}\left(\frac{d}{d t}\right)^{n} \int_{a}^{t} \frac{x(\tau)}{(t-\tau)^{1+\alpha-n}} d \tau \quad \text { a.e }(a, b],
\end{aligned}
$$

where $\Gamma(\alpha)$ is the Euler Gamma function. The above integrals are called (respectively) left and right Riemann-Liouville fractional integral.

The Caputo fractional derivative for a ( $n$ time differentiable) function $x(t)$ is defined by

$$
{ }^{C} D_{a^{+}}^{\alpha} x(t)=\frac{1}{\Gamma(1-\alpha)} \int_{a}^{t} \frac{x^{(n)}(\tau)}{(t-\tau)^{1+\alpha-n}} d t \quad \text { a.e }(a, b] .
$$


Proposition 1. Suppose $\alpha \in(n-1, n]$ where $n \in \mathbb{N}$ and assume $m \in \mathbb{N}$. Then

$$
\begin{aligned}
D_{a^{+}}^{\alpha} I_{a^{+}}^{\alpha} x(t) & =x(t), \\
{ }^{C} D_{a^{+}}^{\alpha} I_{a^{+}}^{\alpha} x(t) & =x(t), \\
I_{a^{+}}^{\alpha} D_{a^{+}}^{\alpha} x(t) & =x(t)-\sum_{k=1}^{n} \frac{x^{\alpha-k}}{\Gamma(\alpha-k+1)} D_{a^{+}}^{\alpha-k} x\left(a^{+}\right), \\
D^{m} D_{a^{+}}^{m-\alpha} x(t) & =D_{a^{+}}^{\alpha} x(t), \\
I_{a^{+}}^{\alpha}{ }^{C} D_{a^{+}}^{\alpha} x(t) & =x(t)-\sum_{k=1}^{n} \frac{x^{k}}{k !} x^{(k)}\left(a^{+}\right) .
\end{aligned}
$$

Here, $D_{a^{+}}^{\alpha-k} x\left(a^{+}\right)$and $x^{(a)}\left(a^{+}\right)$are defined by

$$
D_{a^{+}}^{\alpha-k} x\left(a^{+}\right)=\lim _{t \rightarrow a^{+}} D_{a^{+}}^{\alpha-k} x(t), \quad x^{(k)}\left(a^{+}\right)=\lim _{t \rightarrow a^{+}} x^{(k)}(t) .
$$

\section{On a perception of initial values}

As it is mentioned in [11], the 300 years old problem of a physical and geometrical interpretations of integration and differentiation of an arbitrary real order has been listed as one of the open problems at the first international conference of the fractional calculus in New Haven, USA in 1974 [12]. As an examples to some of the reported efforts for the mentioned interpretations see [11, 13].

The same intuitive problem deals with initial values in fractional dynamic problems involving Riemann-Liouville differential and integral operators. Indeed, by a fractional formulation of a real world problem, we expect to get a better description of a mechanism which is presumed to be fit to a proposed fractional model.

It is noteworthy that, in problems involving with a fractional operators in which the ordinary differential operator appears under the integral sign (Caputo or Caputo-Fabrizio D operators), we have the same initial values as they appear in classical problems, i.e., the uniqueness is ensured if a solution $x(t)$ satisfies the initial values $x\left(t_{0}\right)=x_{0}, D x\left(t_{0}\right)=x_{1}, D^{2} x\left(t_{0}\right)=x_{2}, \ldots, D^{n-1} x\left(t_{0}\right)=x_{n-1}$, where $n$ is the order of the equation. In fact, the mentioned fractional differential operators provide this facility to interpret the initial values as initial state, velocity, acceleration and so on.

The corresponding initial values in an IVP involving with Riemann-Liouville DO, sometimes takes a fractional integral form such as

$$
I_{a^{+}}^{1-\alpha} x\left(a^{+}\right)=0
$$

as it appears in (2). The initial values in Riemann-Liouville IVP problems are discussed in [14] and a few interpretations concerning some classes of real world fractional dynamics presented. However, a general interpretation to those mentioned IVs seem to be an open problem. Here our aim is to give an analytical explanation to (14), that facilitates an intuitive way toward a better perception of the problem.

Proposition 2. Suppose $x:[a, b] \rightarrow \mathbb{R}$ possesses fractional integral of order $1-\alpha$ and assume further that, $x$ is analytic on at least a small neighborhood of $t=a$, namely on $(a-\epsilon, a+\epsilon)$ for some positive $\epsilon$. Then $I_{a^{+}}^{1-\alpha} x\left(a^{+}\right)=0$.

Proof. See [9], section 7.2.5.

Remark 1. The initial condition (14) is satisfied even under a weaker criteria than that of stated in Proposition 2.1: 
1) Let $x(t)$ be differentiable on $[a, \epsilon)$ (for some positive $\epsilon$ ) with bounded derivative. Then we have (14). Indeed, differentiability on $[a, a+\epsilon)$, provides integrating by parts

$$
\int_{a}^{t} \frac{x(\tau) d \tau}{(t-\tau)^{\alpha}}=\frac{x_{a}(t-a)^{1-\alpha}}{1-\alpha}+\frac{1}{1-\alpha} \int_{a}^{t} x^{\prime}(\tau)(t-\tau)^{1-\alpha} d \tau, \quad t \in[a, a+\epsilon)
$$

and (14) is obtained while $t \rightarrow a^{+}$.

2) Assume that, $x$ is continuous on $[a, a+\epsilon$ ), (for some positive $\epsilon$ ) then by mean value theorem, we have

$$
I_{a^{+}}^{1-\alpha} x\left(a^{+}\right)=x(\xi(t)) \cdot \frac{(t-a)^{1-\alpha}}{\Gamma(2-\alpha)}, \quad \xi(t) \in[a, a+\epsilon)
$$

and we obtain (14) when $t \rightarrow a^{+}$.

Let $\epsilon$ be positive so that $a+\epsilon<b$. we denote $\delta(t)$ to be a function defined by

$$
\delta(t)=\sup _{s \in[a, t]} I_{a^{+}}^{1-\alpha} x(s) .
$$

It is clear that, $\delta$ is continuous on $[a, a+\epsilon)$ for $\epsilon$ small enough. Moreover, it is increasing so, it is differentiable on $(a, a+\epsilon)$.

Proposition 3. Suppose $x:[a, b] \rightarrow \mathbb{R}$ is fractional differentiable of order $\alpha$ and assume that, it satisfies initial conditions $x(a)=x_{a}$ and (14). Then

$$
x_{a} \leq \delta^{\prime}\left(a^{+}\right) .
$$

Proof. Since $I_{a^{+}}^{1-\alpha} x(t) \leq \delta(t)$ and $\delta\left(a^{+}\right)=0$, there exists a sub-interval $\left[a, a+\epsilon_{1}\right)$ for which

$$
\frac{d}{d t} I_{a^{+}}^{1-\alpha} x(t) \leq \delta^{\prime}(t), \quad t \in\left[a, a+\epsilon_{1}\right)
$$

otherwise, there must be a sequence $\left\{t_{n}\right\}$ with $t_{n} \rightarrow a^{+}$such that $\left.\frac{d}{d t}\right|_{t=t_{n}} I_{a^{+}}^{1-\alpha} x(t)>\delta^{\prime}\left(t_{n}\right)$. But this contradictory assumption implies that, there exists some $N \in \mathbb{N}$ and a $n \geq N$ so that

$$
\frac{I_{a^{+}}^{1-\alpha} x\left(t_{n}\right)-I_{a^{+}}^{1-\alpha} x\left(t_{n+1}\right)}{t_{n}-t_{n+1}}>\frac{\delta\left(t_{n}\right)-\delta_{t_{n+1}}}{t_{n}-t_{n+1}}
$$

and we infer that

$$
I_{a^{+}}^{1-\alpha} x\left(t_{n+1}\right)-\delta\left(t_{n+1}\right)<I_{a^{+}}^{1-\alpha} x\left(t_{n}\right)-\delta\left(t_{n}\right) \leq 0,
$$

and we have $I_{a^{+}}^{1-\alpha} x\left(a^{+}\right)<\delta\left(a^{+}\right)$which contradicts the assumptions. now taking a limit $t \rightarrow a^{+}$from (19) for $t \in\left[a, a+\epsilon_{1}\right)$, gives the result.

Remark 2. With the help of the same deduction as in the proof of Proposition 2.3, one can prove the statement below

Let $f, g:(a, b) \rightarrow \mathbb{R}$ be fractional differentiable of order $\alpha$. If $I_{a^{+}}^{1-\alpha} f\left(a^{+}\right)=I_{a^{+}}^{1-\alpha} g\left(a^{+}\right)$and $f \leq g$ on a sub-interval $(a, a+\epsilon)$ (for some $0<\epsilon$ ), then $D_{a^{+}}^{\alpha} f(t) \leq D_{a^{+}}^{\alpha} g(t)$ for $t \in\left(a, a+\epsilon_{1}\right)$ where $0<\epsilon_{1} \leq \epsilon$.

If we assume that, $\delta$ is absolutely continuous on $[a, a+\epsilon]$, then $\delta_{\alpha}=I_{a^{+}}^{\alpha} \delta$ is absolutely continuous. This is equivalent to the existence of $D_{a^{+}}^{1-\alpha} \delta(t)$.

Suppose $f, g:[a, b] \rightarrow \mathbb{R}$ and let

$$
\lim _{t \rightarrow a^{+}}(f(t)-g(t))=0 .
$$


It is easy to verify that

$$
\lim _{t \rightarrow a^{+}} I_{a^{+}}^{\alpha}(f(t)-g(t))(t)=0
$$

Taking $f(t)=I_{a^{+}}^{1-\alpha} x(t)$ and $g(t)=\delta(t)$, we conclude that, $I_{a^{+}}^{\alpha} I_{a^{+}}^{1-\alpha} x\left(a^{+}\right)=I_{a^{+}}^{\alpha} \delta\left(a^{+}\right)$. Since we have $I_{a^{+}}^{1-\alpha} x(t) \leq \delta(t)$, then $I_{a^{+}}^{\alpha} I_{a^{+}}^{1-\alpha} x(t) \leq I_{a^{+}}^{\alpha} \delta(t)$ holds for $t \in[a, a+\epsilon)$. Using the proof of Proposition 3 , we obtain

$$
\begin{aligned}
x(t)=\frac{d}{d t} I^{1} x(t) & =\frac{d}{d t} I_{a^{+}}^{\alpha} I_{a^{+}}^{1-\alpha} x(t) \\
& \leq \frac{d}{d t} I_{a^{+}}^{\alpha} \delta(t) \\
& =D_{a^{+}}^{1-\alpha} \delta(t), \quad t \in\left[a, a+\epsilon_{1}\right)
\end{aligned}
$$

for some $0<\epsilon_{1} \leq \epsilon$. Specially, by taking a limit $t \rightarrow a^{+}$, we have $x_{a} \leq D_{a^{+}}^{1-\alpha} \delta\left(a^{+}\right)$and we have proved the statement below.

Proposition 4. Suppose $x:[a, b] \rightarrow \mathbb{R}$ is fractional differentiable of order $\alpha$ and assume that, it satisfies initial conditions $x(a)=x_{a}$ and (14). Then

$$
x_{a} \leq D_{a^{+}}^{1-\alpha} \delta\left(a^{+}\right)
$$

provided $\delta$ is absolutely continuous and it is defined by (17).

In the next section, we convert the the problem (2) into an ordinary initial value problem (involving ordinary integro-differential operators).

\section{A Converted System}

To convert the system (2) into an integral equation we use an auxiliary variable introduced in [15] by which, systems involving the Riemann-Liouville differential operator is converted into systems containing the Caputo differential operator. First, the existence of such auxiliary variable should be clarified: Let $\mathcal{C}$ denotes the class of functions $\xi \in C^{1}(a, b)$ with bounded derivative, i.e., $\left\|\xi^{\prime}\right\|_{\infty}=$ $\sup _{t \in(a, b)}\left|\xi^{\prime}(t)\right|<\infty$. It is immediate by the mean value theorem that, the following identity holds for $t \in(a, b)$

$$
{ }^{C} D_{a^{+}}^{\alpha} \xi(t)=\frac{\xi^{\prime}\left(t_{0}\right)}{\Gamma(2-\alpha)},
$$

for some $t_{0} \in(a, t)$; then, for $\xi \in \mathcal{C}$

$$
{ }^{C} D_{a^{+}}^{\alpha} \xi\left(a^{+}\right)=\lim _{t \rightarrow a^{+}}{ }^{C} D_{a^{+}}^{\alpha} \xi(t)=0,
$$

and we have the following Lemma.

Lemma 1. Suppose $x(t)$ be such that $I_{a^{+}}^{1-\alpha} x(t)$ exists for $t \in[a, b]$ and $I_{a^{+}}^{\alpha} x\left(a^{+}\right)=0$. Then there exists some $\xi \in \mathcal{C}$ for which

$$
x(t)={ }^{C} D_{a^{+}}^{1-\alpha} \xi(t)+x(a) .
$$

Proof. It is easy to verify that, the functions

$$
\xi(t)=I_{a^{+}}^{1-\alpha} x(t)-\frac{x(a)(t-a)^{1-\alpha}}{\Gamma(2-\alpha)}+\xi_{a} \quad \xi_{a}, \in \mathbb{R},
$$

satisfy (28). 
Now suppose $x(t)$ is the optimal state which satisfies (1) and extremizes (2), so it satisfies the conditions of Lemma 1.1. By the definition of fractional derivatives and fractional integral and some primitive properties (see for example [10]) we have

$$
\begin{aligned}
D_{a^{+}}^{\alpha} x(t)=D I_{a^{+}}^{1-\alpha} x(t) & =D I_{a^{+}}^{1-\alpha}\left({ }^{C} D_{a^{+}}^{1-\alpha} \xi(t)+x_{a}\right) \\
& =D\left(\xi(t)-\xi(a)+\frac{x_{a}(t-a)^{1-\alpha}}{\Gamma(2-\alpha)}\right) \\
& =\xi^{\prime}(t)+\frac{x_{a}(t-a)^{-\alpha}}{\Gamma(1-\alpha)} .
\end{aligned}
$$

Therefore, equation (2) is converted to

$$
\begin{aligned}
\xi^{\prime}(t)=a(t) \cdot{ }^{C} D_{a^{+}}^{1-\alpha} \xi(t)+b(t) u(t) & +\int_{a}^{b} K(t, s) \phi\left({ }^{C} D_{a^{+}}^{1-\alpha} \xi(s)+x_{a}\right) d s \\
& +x_{a} a(t)-\frac{x_{a}(t-a)^{-\alpha}}{\Gamma(1-\alpha)} .
\end{aligned}
$$

According to Pontryagin's optimal control law [16], we need another equation coupled with (31) that is called the co-state equation.

Theorem 2. The fractional optimal control problem (1)-(2) is equivalent to the system

$$
\left\{\begin{aligned}
\xi^{\prime}(t) & =a(t) \cdot{ }^{C} D_{a^{+}}^{1-\alpha} \xi(t)+b(t)\left(\frac{1}{2} g(t)-\lambda(t) b(t)\right) \\
& +\int_{a}^{t} K(t, s) \phi\left({ }^{C} D_{a^{+}}^{1-\alpha} \xi(s)+x_{a}\right) d s+x_{a} a(t)-\frac{x_{a}(t-a)^{-\alpha}}{\Gamma(1-\alpha)} \\
\lambda^{\prime}(t) & =-x_{a}-{ }^{C} D_{a^{+}}^{1-\alpha} \xi(t)-f(t)-\lambda(t) a(t)-\lambda(t) \int_{a}^{t} K(t, s) \phi^{\prime}\left({ }^{C} D_{a^{+}}^{1-\alpha} \xi(s)\right) d s,
\end{aligned}\right.
$$

where $\lambda$ is the co-state variable.

Proof. According to Pontryagin' theory, we have

$$
u^{*}=-\frac{1}{2} g(t)-\lambda(t) b(t)
$$

where $u^{*}$ is the optimal control. Substituting (28) into (2) and the co-state equation, we obtain (32).

\section{Numerical Example}

Let $f(t)=\sin (t), g(t)=\cos (t), a(t)=t+1, b(t)=t, K(t, s)=t-s$ and $\phi(t)=t$, then system (32) is equivalent to

$$
\left\{\begin{array}{l}
\xi(t)=\xi_{a}\left(1-\frac{(t-a)^{\alpha}}{\Gamma(1+\alpha)}\right)+\int_{a}^{t} s I_{a^{+}}^{\alpha} \xi^{\prime}(s) d s+I_{a^{+}}^{\alpha} \xi(t)-\int_{a}^{t} s^{2} \lambda(s) d s+I_{a^{+}}^{2+\alpha} \xi(t) \\
-\frac{(t-a)^{\alpha+2}}{\Gamma(\alpha+2)}+x_{a} \cdot\left(\frac{(t-a)^{3}}{6}+\frac{t^{2}-a^{2}}{2}+t-a-\frac{(t-a)^{1-\alpha}}{\Gamma(2-\alpha)}\right)+\sin (t)+\cos (t)-(a \sin (a)+\cos (a)) \\
\lambda(t)=\lambda_{a}-x_{a} \cdot(t-a)-I_{a^{+}}^{\alpha} \xi(t)+\xi_{a} \cdot \frac{(t-a)^{\alpha}}{\Gamma(\alpha+1)}-\int_{a}^{t}\left(\frac{(s-a)^{2}}{2}+s+1\right) \lambda(s) d s+\cos (t)-\cos (a)
\end{array}\right.
$$


The approximate solution of equation (34) using Adomian decomposition method $(A D M)$ [17], with the help of Mathematica for $\alpha=\frac{2}{3}, \xi_{a}=0, x_{a}=1$ and $\lambda_{a}=\beta$, which $\beta \in R$ is an unknown parameter which will be determined later by using boundary conditions, we find the approximate solution given by

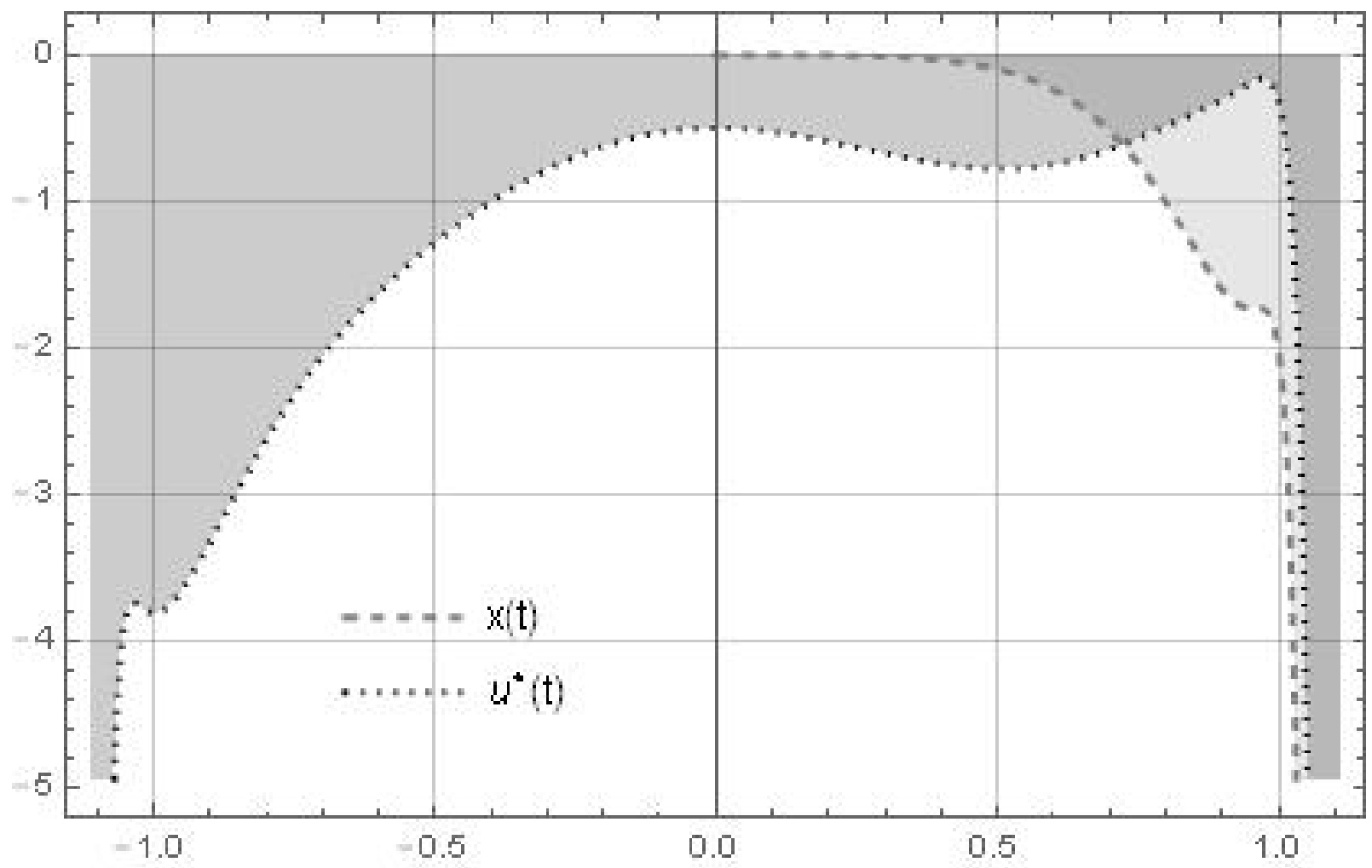

Fig. 1: The approximate solution in the case $\alpha=\frac{2}{3}$ for Eqs. (37) and (38).

$$
\begin{aligned}
\xi(t) \approx & \frac{t^{12}}{36 \Gamma\left(\frac{5}{3}\right)}+t^{4}\left(-\frac{1}{2}+\frac{387296 \Gamma\left(\frac{4}{3}\right)}{2187 \Gamma\left(\frac{22}{3}\right)}+\frac{7 \pi}{729 \Gamma\left(\frac{2}{3}\right) \Gamma\left(\frac{4}{3}\right)}\right)+ \\
& t^{16}\left(-\frac{13832 \Gamma\left(\frac{4}{3}\right)^{2}}{15 \pi \Gamma\left(\frac{22}{3}\right)}-\frac{27}{20 \Gamma\left(\frac{2}{3}\right)}\right)+t^{15}\left(\frac{110656 \Gamma\left(\frac{4}{3}\right)^{2}}{45 \pi \Gamma\left(\frac{22}{3}\right)}+\frac{18}{5 \Gamma\left(\frac{2}{3}\right)}\right)+ \\
& \frac{t^{10}}{4 \Gamma\left(\frac{5}{3}\right)}+t^{2}\left(-4 \sin (t)+2 \cos (t)+\frac{933}{104720 \Gamma\left(\frac{2}{3}\right)}\right)+t(-4 \sin (t)-8 \cos (t))+ \\
& 8 \sin (t)+t^{6}\left(\frac{2 \cos (t)}{\Gamma\left(\frac{5}{3}\right)}-\frac{4 \sin (t)}{\Gamma\left(\frac{5}{3}\right)}-\frac{1}{18}-\frac{2}{\Gamma\left(\frac{5}{3}\right)}\right)+t^{9}\left(\frac{B}{3 \Gamma\left(\frac{5}{3}\right)}-\frac{2}{3 \Gamma\left(\frac{5}{3}\right)}\right)+ \\
& t^{8}\left(-\frac{\cos (t)}{\Gamma\left(\frac{5}{3}\right)}+\frac{2 \sin (t)}{\Gamma\left(\frac{5}{3}\right)}+\frac{37503}{61600 \Gamma\left(\frac{2}{3}\right)}\right)+t^{7}\left(\frac{4 \cos (t)}{\Gamma\left(\frac{5}{3}\right)}+\frac{2 \sin (t)}{\Gamma\left(\frac{5}{3}\right)}-\frac{18}{5 \Gamma\left(\frac{2}{3}\right)}\right)
\end{aligned}
$$


and

$$
\begin{aligned}
\lambda(t) \approx & \frac{4293 t^{18}}{143990 \Gamma\left(\frac{2}{3}\right)}+t^{3}\left(-\frac{B}{6}+\frac{1}{6}-\frac{2}{\Gamma\left(\frac{4}{3}\right)}\right)+t^{4}\left(-\frac{7}{24}-\frac{7 \pi}{729 \Gamma\left(\frac{2}{3}\right) \Gamma\left(\frac{4}{3}\right)}\right)+ \\
& t^{9}\left(\frac{2}{3 \Gamma\left(\frac{5}{3}\right)}-\frac{B}{3 \Gamma\left(\frac{5}{3}\right)}\right)+t^{6}\left(-\frac{2 \cos (t)}{\Gamma\left(\frac{5}{3}\right)}+\frac{4 \sin (t)}{\Gamma\left(\frac{5}{3}\right)}-\frac{1}{72}+\frac{2}{\Gamma\left(\frac{5}{3}\right)}\right) \\
& t^{12}\left(\frac{1}{\Gamma\left(\frac{7}{3}\right)}-\frac{1}{36 \Gamma\left(\frac{5}{3}\right)}\right)-\frac{2 t^{24}}{\Gamma\left(\frac{8}{3}\right)}+t(-B-3 \sin (t)-\cos (t)+3)+ \\
& t^{2}\left(-\frac{B}{2}-\sin (t)+\frac{\cos (t)}{2}+\frac{3}{2}\right)+\sin (t)+t^{7}\left(-\frac{4 \cos (t)}{\Gamma\left(\frac{5}{3}\right)}-\frac{2 \sin (t)}{\Gamma\left(\frac{5}{3}\right)}+\frac{18}{5 \Gamma\left(\frac{2}{3}\right)}\right)+ \\
& t^{8}\left(\frac{\cos (t)}{\Gamma\left(\frac{5}{3}\right)}-\frac{2 \sin (t)}{\Gamma\left(\frac{5}{3}\right)}-\frac{37503}{61600 \Gamma\left(\frac{2}{3}\right)}\right)-\frac{t^{10}}{4 \Gamma\left(\frac{5}{3}\right)}-\frac{t^{11}}{10 \Gamma\left(\frac{5}{3}\right)}
\end{aligned}
$$

Know, with regarding to Eq.(28), we have:

$$
\begin{aligned}
x(t) \approx & \frac{1}{\Gamma\left(\frac{2}{3}\right)}\left(\frac{7 \sqrt{3}}{220}-\frac{3483}{2200}\right) t^{11 / 3}+\left(\frac{243}{220}+\frac{3 \sqrt{3}}{44}\right) t^{14 / 3}-\frac{729 t^{17 / 3}}{5236}+ \\
& \frac{t^{56 / 3} 53922 \Gamma\left(\frac{4}{3}\right)}{\pi 43943 \Gamma\left(\frac{2}{3}\right)}-\frac{t^{59 / 3} 50031 \Gamma\left(\frac{4}{3}\right)}{\pi 17880 \Gamma\left(\frac{2}{3}\right)}+\frac{933 t^{5 / 3}}{52360 \Gamma\left(\frac{2}{3}\right) \Gamma\left(\frac{8}{3}\right)}- \\
& \frac{18144 t^{20 / 3}}{\Gamma\left(\frac{2}{3}\right) \Gamma\left(\frac{23}{3}\right)}+\frac{t^{23 / 3} 13501}{55 \Gamma\left(\frac{2}{3}\right) \Gamma\left(\frac{26}{3}\right)}+\frac{t^{29 / 3} 22680}{\Gamma\left(\frac{2}{3}\right) \Gamma\left(\frac{32}{3}\right)}- \\
& \frac{t^{32 / 3} 13970}{\Gamma\left(\frac{2}{3}\right) \Gamma\left(\frac{35}{3}\right)}+\frac{t^{35 / 3} 19958}{\Gamma\left(\frac{2}{3}\right) \Gamma\left(\frac{38}{3}\right)} .
\end{aligned}
$$

Also, with Eq.(33) optimal control will be

$$
\begin{aligned}
u^{*}(t) \approx & \frac{2 t^{10}}{3 \Gamma\left(\frac{5}{3}\right)}+\frac{t^{11}}{4 \Gamma\left(\frac{5}{3}\right)}+\frac{t^{12}}{10 \Gamma\left(\frac{5}{3}\right)}+t^{13}\left(\frac{1}{36 \Gamma\left(\frac{5}{3}\right)}-\frac{1}{\Gamma\left(\frac{7}{3}\right)}\right)+ \\
& t^{9}\left(-\frac{\cos (t)}{\Gamma\left(\frac{5}{3}\right)}+\frac{2 \sin (t)}{\Gamma\left(\frac{5}{3}\right)}+\frac{37503}{61600 \Gamma\left(\frac{2}{3}\right)}\right)+t^{2}(3 \sin (t)+\cos (t)-3)+ \\
& t^{7}\left(\frac{2 \cos (t)}{\Gamma\left(\frac{5}{3}\right)}-\frac{4 \sin (t)}{\Gamma\left(\frac{5}{3}\right)}+\frac{1}{72}-\frac{2}{\Gamma\left(\frac{5}{3}\right)}\right)+t^{3}\left(\sin (t)-\frac{\cos (t)}{2}-\frac{3}{2}\right)+ \\
& t^{8}\left(\frac{4 \cos (t)}{\Gamma\left(\frac{5}{3}\right)}+\frac{2 \sin (t)}{\Gamma\left(\frac{5}{3}\right)}-\frac{18}{5 \Gamma\left(\frac{2}{3}\right)}\right)+t(-\sin (t)-2 \cos (t)+2)
\end{aligned}
$$

The plot in Fig.1, shows the approximate solution expressed by (37) and (38) when $\alpha=\frac{2}{3}$.

\section{Conclusion}

In the present work, using the ADM method. an approximate solution to an extremization problem subject to a Riemann-Liouville initial value problem is illustrated. In the subsection 2.1, the initial 
value (14) which is given in the constraint (2) is studied and necessary and sufficient conditions for (14) are obtained.

Making use of the suitable change of variable in section 2, provided a conversion of the original FOC problem into the system (32).

\section{References}

[1] K.H. Maleknejad, A. Ebrahimzadeh, Optimal control of volterra integro-differential systems based on Legendre wavelets and collocation method, International Journal of Mathematical, Computational, Physical, Electrical and Computer Engineering. 8(7) (2014) 1040-1044.

[2] V.V. Uchaikin, Fractional derivatives for physicists and engineers, Springer, Berlin, 2013.

[3] D. Baleanu et al., Fractional caculus, models and numerical methods, World Scientific, 2012.

[4] V.E. Tarasov, Review of some promising fractional physical models, International Journal of Modern Physics B. 27(9) (2013).

[5] W. Chen et al., Anomalous diffusion modeling by fractal and fractional derivatives, Computers and Mathematics with Applications. 59(5) (2010) 1754-1758.

[6] R. Metzler, W.G. Glöckle, T.F. Nonnenmacher, Fractional model equation for anomalous diffusion, Physica A: Statistical Mechanics and its Applications. 211(1) (1994) 13-24.

[7] R. Almeida, D.F. Torres, A discrete method to solve fractional optimal control problems, Nonlinear Dynamics. 80(4) (2015) 1811-1816.

[8] E. Ghomanjani, A numerical technique for solving fractional optimal control problems and fractional Riccati differential equations, Journal of the Egyptian Mathematical Society. 24(4) (2015) 638-643.

[9] I. Podlubny, Fractional differential equations, Academic Press, 1999.

[10] S.G. Samko, A.A. Kilbas, O.I. Marichev, Fractional integrals and derivatives, theory and applications, Gordon and Breach Science Publishers, 1993.

[11] I. Podlubny, Geometric and physical interpretations of fractional integration and fractional differentiation, Fractional Calculus and Applied Analysis. 5(4) (2002) 367-386.

[12] B. Ross, Fractional calculus and its applications, Lecture Notes in Mathematics, vol. 457, Springer-Verlag, New York, 1975.

[13] J.F. Gomez-Aguilar, R. Razo-Hern'andez, D. Granados-Lieberman, A physical interpretation of fractional calculus in observables terms: analysis of the fractional time constant and the transitory response, Revista Mexicana de F'1sica. 60 (2014) 32-38.

[14] N. Heymann, I. Podlubny, Physical interpretation of initial conditions for fractional differential equations with Riemann-Liouville fractional derivatives, Rheologica Acta. 45(5) (2006) 765-771.

[15] V.E. Tarasov, Fractional dynamics, Applications of fractional claculus to dynamics of particles fields and media, Springer Verlag, 2010.

[16] I.M. Ross, A primer on Pontryagin's principle in optimal control, Collegiate Publishers, 2015.

[17] G. Adomian, Solving frontier problems of physics: the decomposition method, Vol. 60, Springer Science \& Business Media, 2013. 